\title{
Resección de un liposarcoma retroperitoneal gigante: a propósito de un caso
}

Begliardo FL, Corpacci M, Obelar-Briones MF, García R, Bender AL

Resumen

ANTECEDENTES: los liposarcomas son los tumores sarcomatoides de partes blandas más comunes en los adultos. Los sitios más frecuentes de aparición son las extremidades, el retroperitoneo y la región inguinal.

CASO CLÍNICO: paciente masculino de 57 años de edad, quien consulta por una masa abdominal, de crecimiento progresivo, de 8 meses de evolución. La tomografía computada objetivó una masa originada a partir del retroperitoneo, que encapsulaba el riñón derecho y desplazaba los órganos abdominales hacia la izquierda. Mediante laparotomía exploradora se logró la exéresis completa del tumor retroperitoneal. El informe anatomopatológico definitivo fue de liposarcoma bien diferenciado, con márgenes quirúrgicos negativos (R0) y tumor de $56 \times 35 \times 30 \mathrm{~cm}$, de $27 \mathrm{~kg}$.

CONCLUSIÓN: la resección completa de los liposarcomas representa la única posibilidad de tratamiento radical, lo que sugiere el factor predictivo más importante de recurrencia y supervivencia.

PALABRAS CLAVES: liposarcoma; neoplasias retroperitoneales; sarcoma.

Rev Mex Urol. 2017 Nov-Dec;77(6):481-485.

\section{Resection of a giant retroperitoneal liposarcoma: A case report}

Begliardo FL, Corpacci M, Obelar-Briones MF, García R, Bender AL

\begin{abstract}
BACKGROUND: Liposarcoma is the most common soft tissue sarcoma in adults. Its most frequent presentation sites are the limbs, the retroperitoneum, and the inguinal region.

CLINICAL CASE: A 57-year-old man sought medical attention for a continually growing abdominal mass of 8-month progression. Computed tomography revealed a mass in the retroperitoneum, encapsulating the right kidney, and displacing the abdominal organs to the left. During exploratory laparotomy, the retroperitoneal tumor was
\end{abstract}

Servicio de Cirugía General, Nuevo Hospital San Roque, Córdoba, República de Argentina.

Recibido: septiembre 2017

Aceptado: octubre 2017

Correspondencia

Fernando Luis Begliardo

ferbegliardo@hotmail.com

Este artículo debe citarse como

Begliardo FL, Corpacci M, Obelar-Briones MF, García $\mathrm{R}$, Bender AL. Resección de un liposarcoma retroperitoneal gigante: a propósito de un caso. Rev Mex Urol. 2017 nov-dic;77(6):481-485.

DOI: https://doi.org/10.24245/revmexurol.v77i6.1642 
completely excised and the definitive anatomopathologic study stated well differentiated liposarcoma, with negative surgical margins (R0), measuring $56 \times 35 \times 30 \mathrm{~cm}$ and weighing $27 \mathrm{~kg}$.

CONCLUSION: Currently, the only radical treatment is complete resection and it is the most important predictive factor of recurrence and survival.

KEYWORDS: Liposarcoma; Retroperitoneal neoplasias; Sarcoma
Servicio de Cirugía General, Nuevo Hospital San Roque, Córdoba, República de Argentina.

Correspondence

Fernando Luis Begliardo

ferbegliardo@hotmail.com

\section{ANTECEDENTES}

Los liposarcomas son los tumores sarcomatoides de partes blandas más comunes en los adultos. Los sitios más frecuentes de aparición incluyen las extremidades, el retroperitoneo y la región inguinal. ${ }^{1}$ Suelen afectar a pacientes de entre 40 y 60 años de edad, sin importar el sexo. ${ }^{2}$ Los liposarcomas retroperitoneales son difíciles de diagnosticar tempranamente, pues sus síntomas son inespecíficos y de aparición tardía.

Los liposarcomas pueden tener peso y dimensión variables; por ejemplo, los mayores de 20 kg son Ilamados "liposarcomas gigantes" y son extremadamente raros. ${ }^{2}$

\section{CASO CLÍNICO}

Paciente masculino de 57 años de edad, quien acudió a consulta por distensión abdominal de inicio insidioso y aumento progresivo de 8 meses de evolución. Entre sus antecedentes personales refirió hipertensión arterial, cardiopatía chagásica, diabetes, hipotiroidismo y asma. En el examen físico se observó el abdomen distendido y se palpó una masa de consistencia indurada, no dolorosa.

Los estudios de laboratorios resultaron sin alteraciones. La tomografía computada con contraste endovenoso y oral objetivó una gran masa originada a partir del retroperitoneo, que encapsulaba el riñón derecho y desplazaba los órganos abdominales hacia el flanco izquierdo (Figura 1). La arteriografía renal descartó obstrucciones significativas. Aunque los estudios de imagen preoperatorios sugerían la coexistencia de un sarcoma retroperitoneal y con la finalidad de descartar alguna alteración adicional que cambiara la conducta quirúrgica, se decidió la aspiración con aguja fina ecodirigida en dicha tumoración.

Con anestesia general se llevó a cabo la laparotomía exploradora, que evidenció un tumor retroperitoneal, polilobulado, caracterizado por la coexistencia de áreas de diferente densidad, sin necrosis. Durante la resección de los distintos segmentos del tumor se identificó el riñón derecho ptósico. Se decidió la nefrectomía derecha, pues la masa envolvía completamente el riñón y dificultaba encontrar un plano libre de separación. Posteriormente se logró la exéresis radical de toda la tumoración retroperitoneal.

El paciente ingresó a la unidad de cuidados intensivos, en la que permaneció durante tres días; requirió transfusión de 3 unidades de glóbulos rojos sedimentados y 5 de plasma fresco congelado. El posoperatorio transcurrió sin cambios hemodinámicos, ni requerimiento de asistencia 


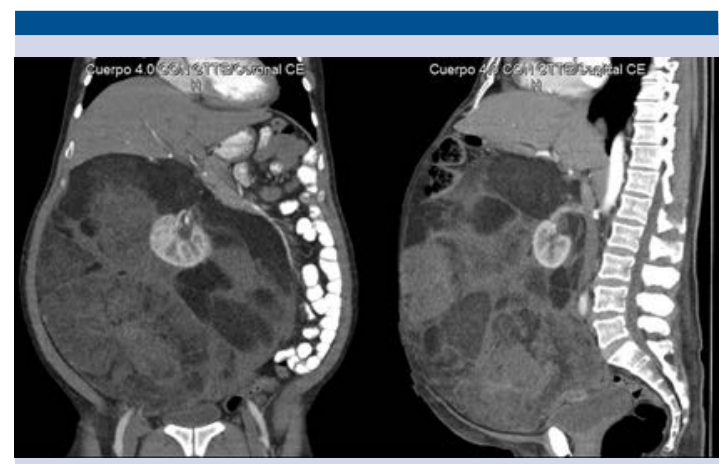

Figura 1. Tomografía computada que evidencia el desplazamiento del contenido abdominal hacia la izquierda, con el riñón derecho encapsulado por el tumor.

mecánica ventilatoria, por lo que se otorgó el alta hospitalaria al quinto día. Hasta el momento (14 meses después de la intervención quirúrgica) no se ha evidenciado recurrencia de la tumoración.

El informe anatomopatológico definitivo fue de liposarcoma bien diferenciado, con márgenes quirúrgicos negativos (R0) y tumor de 56 × $35 \times$ $30 \mathrm{~cm}$, de $27 \mathrm{~kg}$ (Figuras 2 y 3).

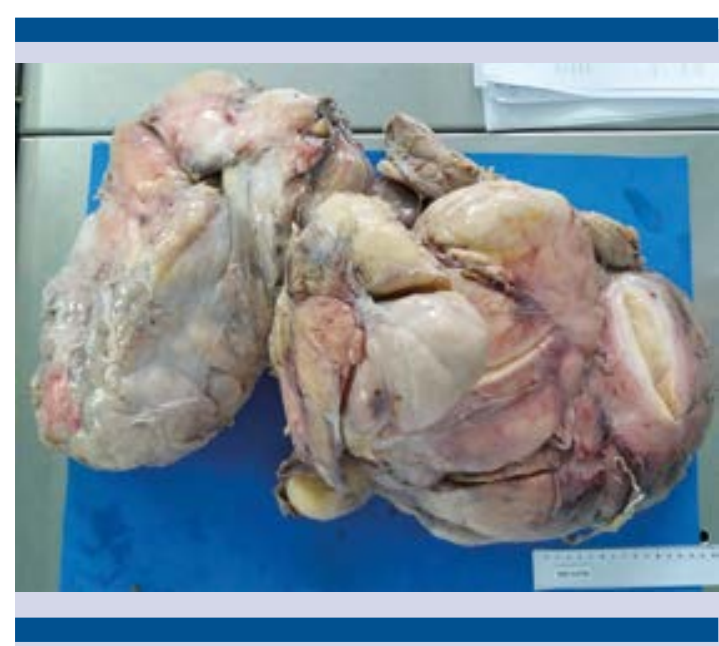

Figura 2. Liposarcoma retroperitoneal (pieza quirúrgica completa, de 56 × 35 × $30 \mathrm{~cm}$ y $27 \mathrm{~kg}$ ).

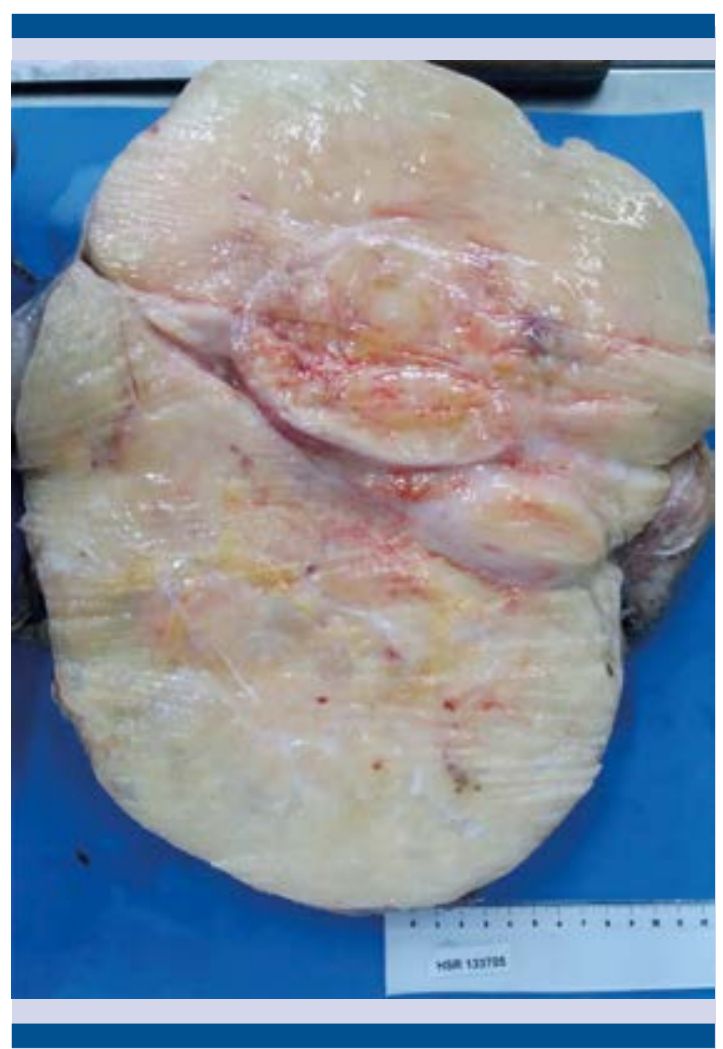

Figura 3. Pieza quirúrgica seccionada.

\section{DISCUSIÓN}

La incidencia de tumores sarcomatoides es relativamente baja (0.8\%). De éstos, el liposarcoma representa $20 \%$ de los casos, principalmente localizado en el retroperitoneo (13\%). ${ }^{3}$

Hasta la fecha se han publicado pocos casos de liposarcoma de gran tamaño en la bibliografía. Algunos reportes señalan tumores de 18, 42 y $46.6 \mathrm{~kg} \cdot{ }^{4-6}$

De acuerdo con la clasificación de la OMS, los liposarcomas se dividen en 5 tipos histológicos: bien diferenciado (46\%), indiferenciado $(18 \%)$, mixoide $(18 \%)$, de células redondas (10\%) y pleomórfico (8\%). El liposarcoma mixoide y el de células redondas se consi- 
deran subtipos, debido a una translocación cromosómica común. ${ }^{1}$ El liposarcoma bien diferenciado tiene buen pronóstico y la supervivencia a 5 años es de $90 \% .^{7}$

La biopsia de los sarcomas retroperitoneales con aguja gruesa es un procedimiento seguro. El diagnóstico histológico preoperatorio es decisivo para diferenciar tumores benignos o malignos de los sarcomas, para diagnosticar los tumores que podrían requerir tratamiento neoadyuvante, y para diagnosticar enfermedad metastásica que se manifiesta como una masa retroperitoneal. Esta técnica se contraindica si la imagen es patognomónica de liposarcoma indiferenciado o, bien, diferenciado y no se planea ningún tratamiento neoadyuvante preoperatorio. $^{8}$

Hoy en día la resección completa representa la única posibilidad de tratamiento radical, lo que sugiere el factor predictivo más importante de recurrencia y supervivencia. Sin embargo, no siempre es fácil obtener márgenes libres del tumor, pues es difícil distinguir la grasa normal del tejido tumoral. Consecuentemente, la recurrencia local es alta $(41 \%){ }^{9}$ Singer y su grupo ${ }^{7}$ reportaron 177 pacientes intervenidos de liposarcoma retroperitoneal, en quienes se registró 73 y $60 \%$ de supervivencia libre de enfermedad a 3 y 5 años, respectivamente.

En algunos casos, para lograr la exéresis quirúrgica completa es necesario realizar la resección de los órganos vecinos afectados, incluso efectuar la nefrectomía en $25 \%$ de los pacientes. ${ }^{10}$ Esta situación no debe ser un impedimento para la cirugía, pues la mortalidad operatoria es baja y la enfermedad local persistente es la principal causa de muerte. ${ }^{11}$

La indicación de terapias multimodales aún se discute. Los liposarcomas de alto grado muestran sensibilidad a la radioterapia; sin embargo, sus efectos tóxicos limitan esta opción como tratamiento primario. ${ }^{2}$ Respecto de la quimioterapia, se han reportado pocas ventajas del tratamiento coadyuvante en pacientes con tumores bien diferenciados de bajo grado y reacciones parciales en los de alto grado (50\%), con incremento en la supervivencia.?

Milone y sus colaboradores ${ }^{12}$ sugieren que el seguimiento continuo durante el posoperatorio es importante para establecer el diagnóstico de recurrencia temprana, con subsecuente cirugía y aumento de la supervivencia.

\section{Conflicto de interés}

Los autores declaran no tener conflictos de interés.

\section{REFERENCIAS}

1. Hashimoto $\mathrm{Y}$, Hatakeyama S, Tachiwada T, et al. Surgical treatment of a giant Liposarcoma in a Japanese man. Adv Urol 2010;2010:943073.

2. Leao P, Vilaca S, Oliveira M, Falcao J. Giant recurrent retroperitoneal liposarcoma initially presenting as inguinal hernia: review of literature. Int J Surg Case Rep 2012;3:103-106.

3. Taki K, Watanabe M, Iwagami S, et al. Giant liposarcoma of the posterior mediastinum and retroperitoneum. BMJ Case Reports 2011. doi: 10.1136/bcr.06.2011.4341.

4. McCallum OJ, Burke JJ, Childs AJ, Ferro A, Gallup DG. Retroperitoneal liposarcoma weighing over one hundred pounds with review of the literature. Gynecol Oncol 2006;103:1152-1154.

5. Yol S, Tavli S, Tavli L, Belviranli M, Yosunkaya A. Retroperitoneal and scrotal giant liposarcoma: report of a case. Surg Today 1998;28:339-342.

6. Herrera-Gómez A, Ortega-Gutiérrez C, Betancourt AM, Luna-Ortiz K. Giant retroperitoneal liposarcoma. World J Surg Oncol 2008;6:115.

7. Singer S, Antonescu CR, Riedel E, Brennan MF. Histologic subtype and margin of resection predict pattern of recurrence and survival for retroperitoneal liposarcoma. Ann Surg 2003;238:52-65.

8. Messiou C, Moskovic E, Vanel D, Morosi C, et al. Primary retroperitoneal soft tissue sarcoma: imaging appearances, pitfalls and diagnostic algorithm. Eur J Surg Oncol 2017;43(7):1191-1198. 
9. Sato Y, Yamamoto S, Fujita S. Retroperitoneal liposarcoma with colonic involvement: a case report. Jpn J Clin Oncol 2014;44(4):374-378.

10. Azpiazu-Arnaiz P, Muro-Bidaurre I, De Frutos-Gamero A, et al. Tumores retroperitoneales. Liposarcoma mixoide retroperitoneal. Presentación de un nuevo caso. Arch Esp de Urol 2000;53(2):170-173.
11. Bevilacqua RG, Rogatko A, Hajdu SI, et al. Prognostic factors in primary retroperitoneal soft-tissue sarcomas. Arch Surg 1991;126:328-334.

12. Milone M, Pezzullo LS, Salvatore G, Pezzullo MG, et al. Management of high-grade retroperitoneal liposarcomas: personal experience. Updates Surg 2011;63:119-124.

\section{AVISO PARA LOS AUTORES}

Revista Mexicana de Urología tiene una nueva plataforma de gestión para envío de artículos: https://www.revisionporpares.com/index.php/RMUrol ahí podrá inscribirse a la base de datos administrada por el sistema Open Journal System (OJS) que ofrece las siguientes ventajas para los autores:

- Subir sus artículos directamente al sistema.

- Conocer, en cualquier momento, el estado de los artículos enviados, es decir, si ya fueron asignados a un revisor, aceptados con o sin cambios, o rechazados.

- Participar en el proceso editorial corrigiendo y modificando sus artículos hasta su aceptación final. 